\title{
Navigation architecture for hypersonic aircraft
}

\author{
Luca Castigliola $^{1}$, Flavia Causa $^{1}$, and Michele Grassi ${ }^{1}$ \\ ${ }^{1}$ Department of Industrial Engineering (DII) - Aerospace Division - University of Naples "Federico \\ II", Naples, Italy
}

\begin{abstract}
This paper aims at presenting an integrated navigation algorithm designed for estimating the navigation state of the STRATOFLY vehicle (LAPCAT-MR3). STRATOFLY project has been funded by the European Commission, under the framework of Horizon 2020 plan, with the aim of assessing the potential of high-speed transport vehicle. The complex interaction between elements of an air-breathing hypersonic vehicle represents a new paradigm in aircraft design. In particular, one of the needs for early GNC analysis in the case of LAPCAT-MR3 vehicle is the assessment of navigation performance over the reference trajectory. The navigation algorithm presented in this paper is based on an augmented state EKF data fusion algorithm exploiting inertial measurements provided by gyroscopes and accelerometers, heading estimates provided by a magnetometer and satellite-based measurements provided by a spaceborne GNSS receiver, considering GPS, GLONASS and GALILEO constellations.
\end{abstract}

\section{Introduction}

STRATOFLY is a European project funded by the European Commission that studies the feasibility of a hypersonic Mach 8 vehicle, flying in the stratosphere at an altitude higher than $30 \mathrm{~km}$ above mean sea level, performing an antipodal civil passenger transport mission. The main purpose of this project is to refine the design and the concept of operations of the LAPCAT-MR2.4, whose conceptual representation is depicted in Fig. 1. Resulted from multiple optimization iterations of the previous LAPCAT-MR1 [1-5], this vehicle is a hypersonic cruiser concept for civil passenger transports flying at Mach 8 and $30 \mathrm{~km}$ altitude.

Commercial civil transport aircraft, flying at subsonic speeds, normally follow a great circle route between the airports of departure and arrival, once they are clear of local traffic. However, supersonic aircrafts are affected by sonic boom that can cause an over-pressure on ground areas. Therefore, preliminary route planning for the LAPCAT-MR2.4 assumes that supersonic flight is only possible over regions of very low population density (oceans and Earth poles). The reference trajectory used to analyse the performance of the integrated navigation algorithm was provided by ESA (European Space Agency) and has been generated using the software ASTOS (AeroSpace Trajectory optimization Software) [6]. The trajectory, depicted in Fig. 2, is divided into four different parts, i.e. flight phases that are: Initial state: the flight plan assumes Brussels airport as departure point, at $0 \mathrm{~km}$ altitude with a departure velocity of $150 \mathrm{~m} / \mathrm{s}$ to ensure lift off. The acceleration on the runway could not be simulated. Furthermore, the initial flight path angle is zero and the heading is $-15 \mathrm{deg}$, i.e. heading slightly west to pass in between the British Islands and Scandinavia. 


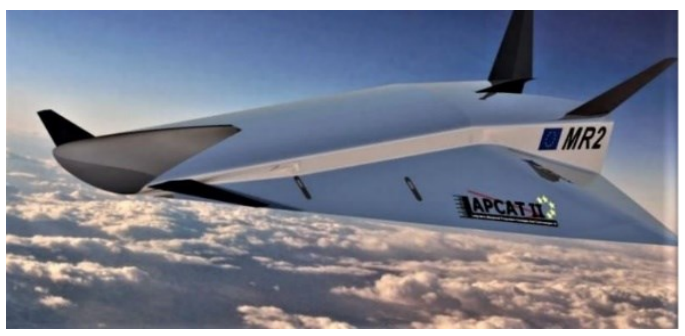

Fig. 1. Rendering of the LAPCAT-MR2.4 [1].

Phase 1: Turbojet ascent. In this stage the ATR (Air-Turbo Rocket) engine is active and a constraint limits the velocity to a Mach number of 0.95 to ensure the subsonic cruise leg. The initial angle of attack is set to 1 degree with bounds ranging from -2 to $2 \mathrm{deg}$.

Phase 2: Turbojet acceleration. In this phase, turbojets accelerate the vehicle up to a Mach number of 4 . The angle of attack has bounds of \pm 2 deg. again and the flight path angle is positive but decreasing because the cruise altitude is about to be reached. At the end of phase 2 , covered distance is $733,4 \mathrm{~km}$.

Phase 3: Ramjet acceleration. This stage is characterised by the switch of the propulsion unit from the ATR to the DMR (Dual Mode Ramjet) engine (ATR deactivated). The acceleration is still positive and stops as soon as Mach 8 is reached. During phase 3 a cruise altitude of almost $36 \mathrm{~km}$ is reached. At the end of this phase, covered distance is $3377,3 \mathrm{~km}$.

Phase 4: cruise to Bering Strait. This is the first part of the cruise. Due to sonic boom requirements, it is necessary to avoid flying over inhabited land and the shortest alternative requires heading north, flying over the arctic region and then passing between Asia and North America at the Bering Strait. At the end of phase 4, covered distance is $6997 \mathrm{~km}$.

Phase 5: cruise to SYD. After the Bering Strait has been passed, flight is being continued with the same constraints as in the phase before. The chosen destination is Sydney and the cruise phase ends at a distance of $15200 \mathrm{~km}$ from the departure target.

Phase 6: descent. The descent to the destination airport is supposed to be an engine-off gliding flight. This means that both propulsion units are deactivated, no fuel will be consumed during this phase and Mach number drops down to subsonic values [6].

This paper aims at introducing a state estimator for the STRATOFLY vehicle. The used sensors and the navigation algorithm are described in sections 2 and 3, respectively. Hence, simulation results on the STRATOFLY nominal trajectory are reported in section 4.

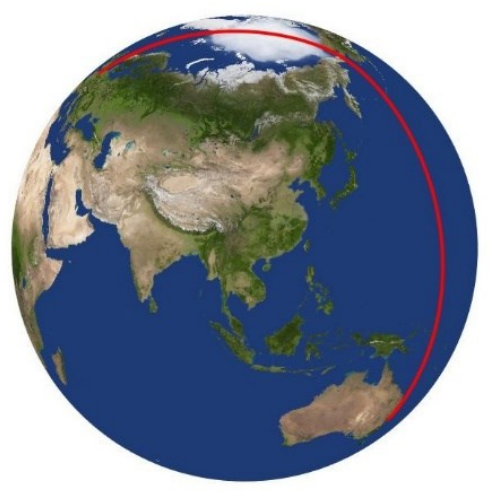

Fig. 2. View on the complete trajectory with pole crossing and Bering Strait passage.

\section{Navigation Sensors}


During the hypersonic and stratospheric cruise, the available instruments to estimate the navigation state are inertial sensors, GNSS receivers, magnetometers and stellar navigation systems. Among these, the navigation algorithm included only inertial sensors and GNSS receivers because the increased accuracy arising from the use of a star-tracker in conjunction with INS and GNSS would have been irrelevant since the LAPCAT-MR2.4 is a large-sized civil aircraft for passenger transport and its missions will never be characterised by aggressive dynamics.

\subsection{Inertial Sensors}

The Inertial Measurement Unit (IMU) taken as reference for this study is the KVH's 1750 IMU, which is a 6 DoF inertial unit exploiting 3 fiber optic gyroscopes and 3 MEMS accelerometers. Its main characteristics are presented in Fig. 3 [7].
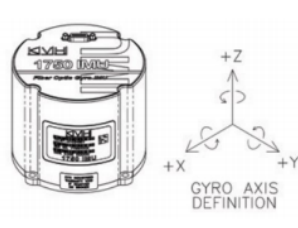

\begin{tabular}{|c|c|c|}
\hline & Gyroscopes (FOG) & Accelerometer (MEMS) \\
\hline Bias Offset & $\pm 2^{\circ} / \mathrm{hr}$ & - \\
\hline Bias Stability & $0.05^{\circ} / \mathrm{hr}, 1 \sigma$ & $0.05 \mathrm{mg}, 1 \sigma$ \\
\hline Scale Factor (non-linearity) & $50 \mathrm{ppm}, 1 \sigma$ & $250 \mathrm{ppm} /{ }^{\circ} \mathrm{C}, 1 \sigma$ \\
\hline ARW / VRW & $0.012^{\circ} / \sqrt{\mathrm{hr}}$ & $0.12 \mathrm{mg} / \sqrt{\mathrm{Hz}}$ \\
\hline
\end{tabular}

Fig. 3. $1750 \mathrm{KVH}$ 's IMU parameters used for the simulation.

\subsection{GNSS Receiver}

The GNSS receiver taken as reference for the implementation and test of the navigation algorithm is in Fig. 4, together with its relevant characteristics [8].

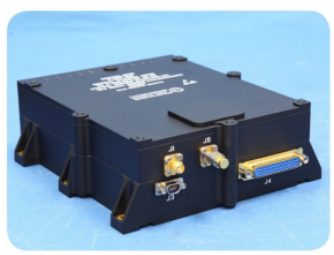

\begin{tabular}{|l|l|}
\hline Frequencies \& codes & L1 \& L2, C/A Code, P(Y), M-Code, L2C optional \\
\hline Pseudorange error & $4.0 \mathrm{~m}, 1 \sigma$ \\
\hline Radiation & $100 \mathrm{Krads}$ \\
\hline Max. velocity & $16000 \mathrm{~m} / \mathrm{s}$ \\
\hline Measurement rate & $1 \mathrm{~Hz}$ \\
\hline
\end{tabular}

Fig. 4. General Dynamics Sentinel M-Code parameters used for the simulation.

The use of GNSS signals in the stratosphere and at hypersonic regimes is not trivial. The first obstacle is represented by COCOM limits: immediate access to satellite measurements and navigation results is disabled when the receiver's velocity is computed to be greater than $1900 \mathrm{~km} / \mathrm{h}(1200 \mathrm{mph})$, or its altitude is computed to be above $18000 \mathrm{~m}(60000 \mathrm{ft})$. This was intended to prevent the use of GPS in intercontinental ballistic missile-like applications. Such a problem can be bypassed in two ways: GNSS receiver's firmware can be "hacked" to disable COCOM limits or space-based GNSS receivers can be considered. For the simulation of the navigation algorithm, the second option was considered because just few manufacturers are allowed to sell COCOM free GNSS receivers, provided that the customer has a proper license; moreover, it would be appropriate to investigate performance degradation of such receivers at higher altitudes and speeds, just like in [9]. In addition, the presence of the plasma sheath generated by the shock-wave at hypersonic speeds is known to generate RF transmission blackout [10]. However, range of altitudes and velocities achieved by the LAPCAT-MR2 during its mission are not sufficient to generate a thick enough plasma sheath around the vehicle, making current hypersonic air-breathing vehicle designs, in general, unlikely to be affected by RF blackout $[11,12]$. 


\section{Kalman Filter}

The navigation algorithm used in this framework is based on a tightly coupled Extended Kalman Filter [13], whose scheme is reported in Fig. 5. The state vector of the EKF $\delta \mathbf{x}$ is:

$$
\delta \mathbf{x}=\left[\delta \phi, \delta \lambda, \delta h, \delta v_{N}, \delta v_{E}, \delta v_{D}, \epsilon_{N}, \epsilon_{E}, \epsilon_{D}, \delta B_{g, x}, \delta B_{g, y}, \delta B_{g, z}, \delta B_{a, x}, \delta B_{a, y}, \delta B_{a, z}\right]^{\mathrm{T}}
$$

where $\phi, \lambda$ and $h$ are respectively latitude, longitude and altitude of the vehicle in the WGS84 model. $v_{N}, v_{E}$ and $v_{D}$ are the components of the vehicle's velocity in the local geographic north-east-down (NED) frame. $\epsilon_{N}, \epsilon_{E}$ and $\epsilon_{D}$ are small-angle rotations defined in NED, indicating the error in computing the rotation between the navigation frame and the body frame (BRF). $B_{g, i}$ and $B_{a, i}$ are the $i$-th component of gyroscope and accelerometer biases in BRF. The filter measurement vector $\delta \mathbf{y}$ is composed by the GPS residuals $\delta \mathbf{y}_{\text {GNSS }}$, the AHRS residuals $\delta \mathbf{y}_{\mathrm{AHRS}}$ and the magnetometer residual $\delta y_{m}$ :

$$
\begin{gathered}
\delta \mathbf{y}=\left[\delta \mathbf{y}_{\mathrm{GNSS}}, \delta \mathbf{y}_{\mathrm{AHRS}}, \delta y_{m}\right]^{\mathrm{T}} \\
\delta \mathbf{y}_{\mathrm{GNSS}}=\left[\delta \rho_{1}-\delta \rho_{n S v}, \ldots, \delta \rho_{n S v-1}-\delta \rho_{n S v}\right] \\
\delta \mathbf{y}_{\mathrm{AHRS}}=\left[\epsilon_{N}, \epsilon_{E}, \epsilon_{D}\right] \\
\delta y_{m}=\psi_{m}-D-\widehat{\psi}
\end{gathered}
$$

The GPS residuals, $\delta \mathbf{y}_{\mathrm{GNSS}}$, are obtained subtracting the pseudorange residual of the pivot satellite, i.e. $\delta \rho_{n S v}$, from the measurement residual of any other satellite, i.e. $\delta \rho_{i}$, where $i=$ $1, \ldots, n S v-1$. This operation is needed to cancel out the receiver clock error. In Eq. 5, $D$ represents the magnetic declination, function of the actual position of the aircraft, $\widehat{\psi}$ is the heading prediction based on inertial measurements and $\psi_{m}$ is the magnetic heading.

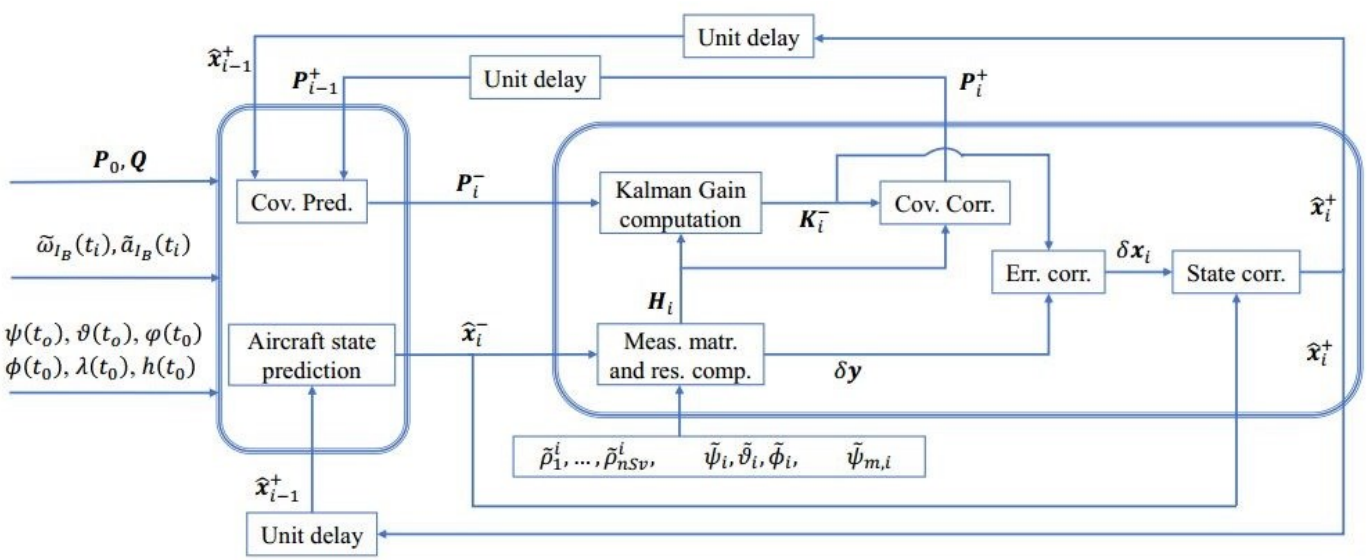

Fig. 5. GNSS range aided INS. $\delta \hat{\mathbf{x}}^{+}$is the a-posteriori estimate of the navigation error; $\hat{\mathbf{x}}^{+}$is the corrected navigation state.

\subsection{Measurement simulation}

The filter has been tested on the nominal STRATOFLY trajectory. Sensor measurements have been simulated based on the nominal trajectory data, using the models described in the following sections.

\subsubsection{Inertial Measurements}


Gyroscope and accelerometer measurements are usually characterized by a gaussian noise, a turn on bias and a bias instability. The latter can be treated as a random walk noise. The measured gyroscope and accelerometer quantities in the body reference frame, i.e. $\widetilde{\boldsymbol{\omega}}_{\mathrm{iB}}^{\mathrm{B}}$ and $\tilde{\mathbf{a}}^{\mathrm{B}}$ respectively, can be thus obtained with:

$$
\begin{gathered}
\widetilde{\boldsymbol{\omega}}_{\mathrm{iB}}^{\mathrm{B}}\left(t_{k}\right)=\boldsymbol{\omega}_{\mathrm{iB}}^{\mathrm{B}}\left(t_{k}\right)+\mathbf{B}_{\mathrm{g}}+\mathbf{N}_{\mathrm{j}}\left(0, \sigma_{g}^{2}\right)+\sum_{\mathrm{i}=1}^{\mathrm{k}} \mathbf{N}_{\mathrm{i}}\left(0, \sigma_{g, n}^{2}\right) \\
\widetilde{\mathbf{a}}^{\mathrm{B}}\left(t_{k}\right)=\mathbf{a}^{\mathrm{B}}\left(t_{k}\right)+\mathbf{B}_{\mathrm{a}}+\mathbf{N}_{\mathrm{j}}\left(0, \sigma_{a}^{2}\right)+\sum_{\mathrm{i}=1}^{\mathrm{k}} \mathbf{N}_{\mathrm{i}}\left(0, \sigma_{a, n}^{2}\right)
\end{gathered}
$$

where $t_{k}$ is the $k$-th instant of time, $\mathbf{B}_{\mathrm{g}}$ and $\mathbf{B}_{\mathrm{a}}$ are the gyroscope and accelerometer bias vectors, $\mathbf{N}_{\mathrm{j}}\left(0, \sigma_{g}^{2}\right)$ and $\mathbf{N}_{\mathrm{j}}\left(0, \sigma_{a}^{2}\right)$ are $3 \times 1$ vectors whose elements are samples extracted from a zero-mean normal distributed stochastic variables with standard deviation $\sigma_{g}=A R W / \sqrt{\delta t}$ ( $A R W$ is the angle random walk) and $\sigma_{a}=V R W / \sqrt{\delta t}$ ( $V R W$ is the velocity random walk). The summation in both equations is the bias stability term, i.e. a random walk process with standard deviation $\sigma_{n}=B S \sqrt{\delta t} / \sqrt{\Delta t}$, where $B S$ is the bias stability parameter specified in the datasheet, and $\Delta t$ is the correlation time.

\subsubsection{GNSS Measurements}

The Matlab GNSS simulator SatNav [14] has been used to simulate GPS, GLONASS and GALILEO constellations: using actual position of the aircraft and day and time of the flight, it is possible to determine all the satellites in view of the aircraft at each epoch, their pseudorange and ECEF position. The simulated pseudoranges include white noise, ionospheric and tropospheric delay, estimated through $[15,16]$ and [17], respectively.

In order to implement LAAS (Local Area Augmented System), a number of differential ground stations was considered, located in Belgium, U.K., Norway and Australia. Differential corrections have been implemented for brief segments of the trajectory, where the ground-station-to-vehicle separation is lower than $100 \mathrm{~km}$. Simulated pseudorange errors measured by the differential ground station are broadcast to the receiver on board the aircraft to remove or reduce pseudorange common errors [18].

The final equation used to model measured pseudorange from the aircraft to the generic $i$-th receiver is Eq. 8, where $R^{i}\left(t_{j}\right)$ is the geometric range from the aircraft to the generic $i-$ th satellite obtained exploiting the tool SatNav, $b$ is the user clock bias, $T$ is the tropospheric delay, $I$ is the ionospheric delay and $v$ is the white noise of the receiver.

$$
\tilde{\rho}^{i}\left(t_{j}\right)=R^{i}\left(t_{j}\right)+c b+c(T+I+v)
$$

\section{Numerical Results}

Fig. 6 shows position errors: both latitude and longitude errors increase dramatically when flying at the North Pole because latitude approaches $90^{\circ}$, and the NED frame is not univocally defined. However, as soon as the vehicle is outside the polar region the algorithm recovers nominal performance and reduces the error of almost three orders of magnitude. The altitude error largely benefits of the bias introduction in the EKF state. Due to the sensors bias drift, indeed, the maximum altitude error reaches almost $200 \mathrm{~m}$, if the IMU biases are not estimated in the filter. Conversely, the addition of $\mathbf{B}_{\mathrm{g}}$ and $\mathbf{B}_{\mathrm{a}}$ to the state vector allows to significantly reduce the altitude error, that reaches a maximum value of $70 \mathrm{~m}$. In Fig. 7, it is also possible to appreciate the benefits of LAAS during departure and landing: during the departure phase 
the error is about $3 \mathrm{~m}$ and jumps to $50 \mathrm{~m}$ as soon as baseline from differential ground station gets larger than the threshold. In addition, even though GDOP is increasing in the landing phase due to a degraded geometry, altitude errors reduce because of the usage of differential measurements.
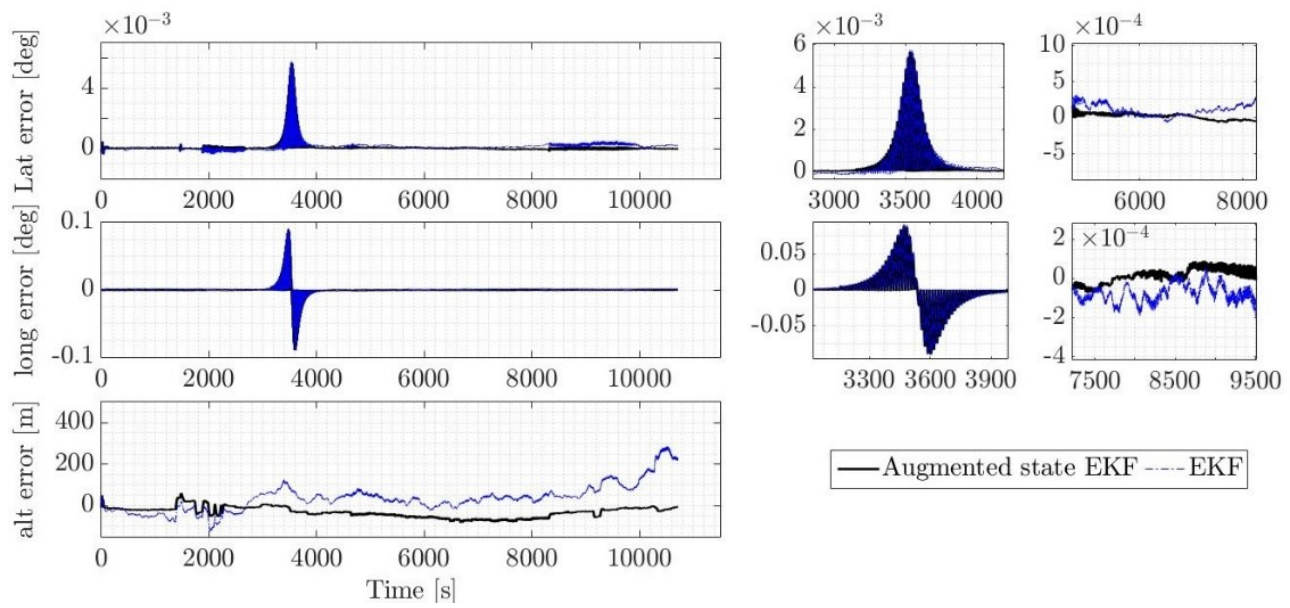

Fig. 6. Estimated position error in terms of latitude, longitude and altitude.

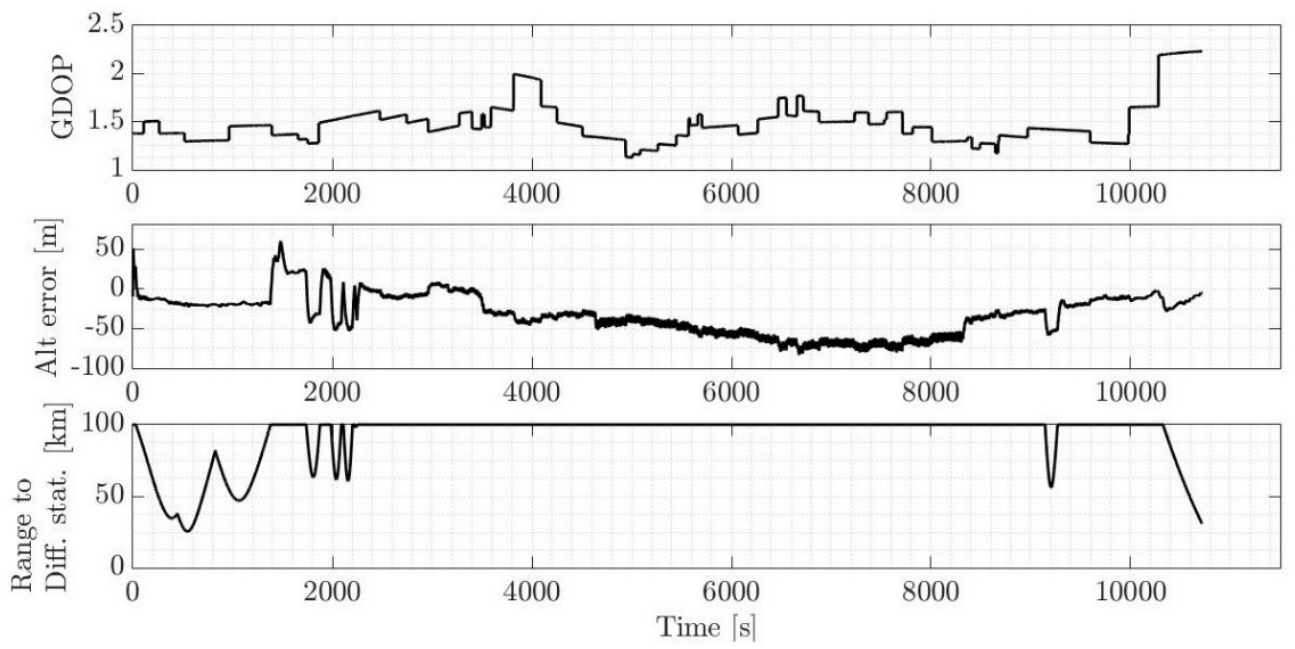

Fig. 7. GDOP along the trajectory (top), estimated altitude error (middle); range to the closest differential station (bottom).

Fig. 8 reports velocity together with velocity errors. Horizontal velocity components present a peak in the error during the polar passages, while vertical velocity errors suffer from inertial sensors bias drift, coherently with altitude error in output from EKF (Fig. 7).

Euler Angles and their estimated errors are in Fig. 9. Heading error increases in the polar region, up to $10^{\circ}$ when using the standard EKF. The benefits of the augmented state EKF on the Euler angles estimation are highlighted in Fig. 9, on the right, where it is apparent that much of the noise, in particular for pitch estimate, has been filtered out. 

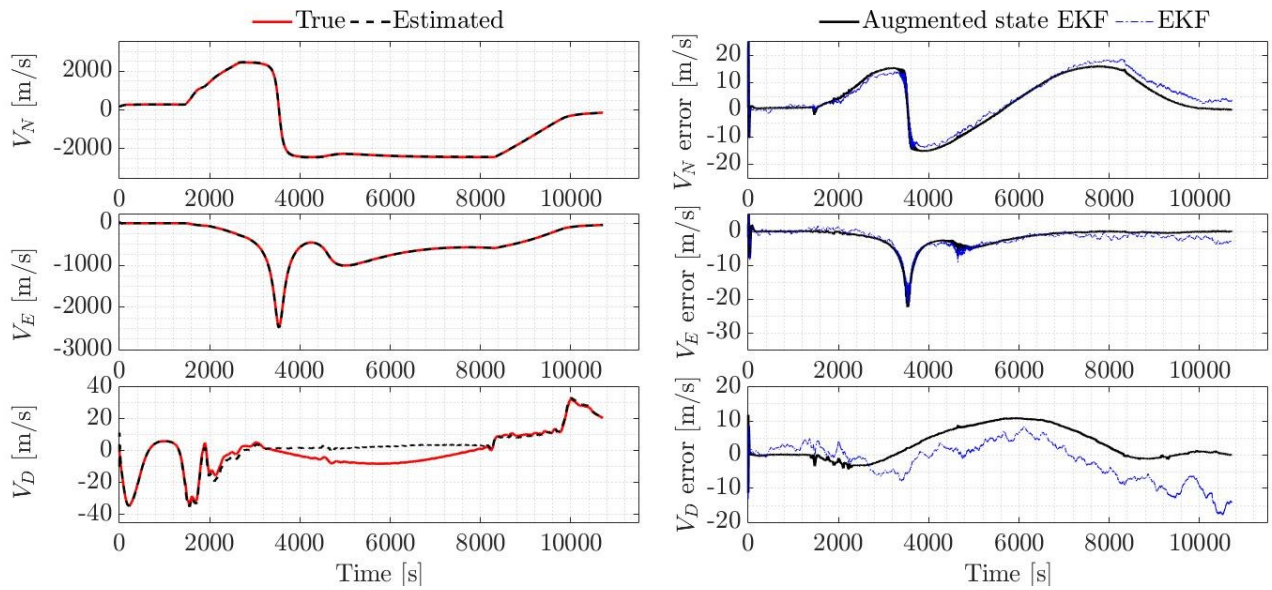

Fig. 8. Comparison between actual and estimated velocity (left); EKF and augmented state estimated velocity error over the whole trajectory (right).
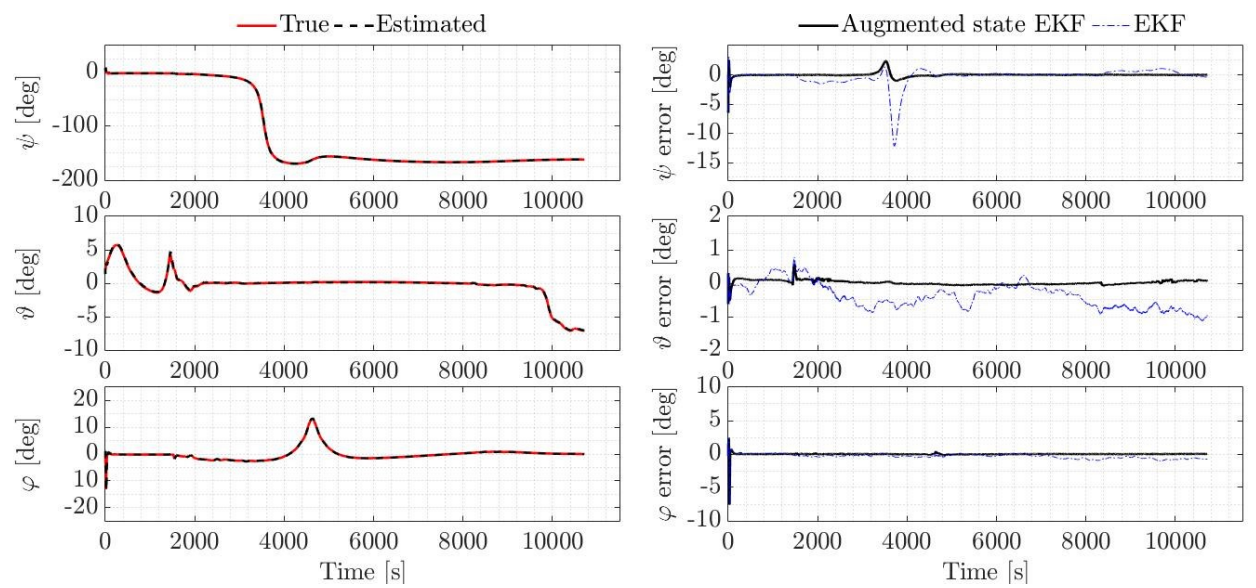

Fig. 9. Comparison between actual and estimated Euler angles (left); EKF and augmented state estimated Euler angles over the whole trajectory.

\section{Conclusions}

This work was aimed at investigating the performance of an INS/GNSS integrated navigation algorithm for a hypersonic aircraft flying in the stratosphere. Main issues were related to the absence of ground navigation aids in the stratosphere, the nearly polar trajectory and COCOM limits for GNSS receivers. Due to the long duration of the trajectory, it was necessary to add to the state vector the gyroscope and the accelerometer biases that allow to model and correct the IMU bias instability. Due to the singularity in the definition of the local frame when approaching the polar region, the navigation error becomes very high with latitudes greater than $85^{\circ}$. Further improvement on the algorithm are required to solve the poles singularity. A common practise includes the application of wander azimuth navigation. However, other strategies are available, e.g. the derivation and usage of the navigation equation in a global frame, like the ECEF frame. 


\section{References}

1. J. Steelant, Advances on Propulsion Technology for High-Speed Aircraft, 12 (2008)

2. J. Steelant, A. Bond, A. Götz, K. Hannemann, C. Bruno, J. Longo, Long-Term Advanced Propulsion Concepts and Technologies (Publishable Final Activity Report, 2008)

3. J. Steelant, AIAA, 17th, AIAA International Space Planes and Hypersonic Systems and Technologies Conference; 2011; San Francisco, CA, 1, pp. 557-564 (2011)

4. J. Steelant, T. Langener, ICAS, $29^{\text {th }}$ International Council of the Aeronautical Sciences, St. Petersburg, Russia; Septmber 7-12, 2014, 29, pp. 173-180 (2014)

5. N. Murray, J. Steelant, A. Mack, ESA SP, 6th, Aerothermodynamics for space vehicles; 2008; Versailles, France, 659, 9 (2009)

6. T. Langener, J. Steelant, Trajectory Analysis for the Updated LAPCAT-MR2.4 Waverider based Vehicle (Publishable Final Activity Report, 2013)

7. https://kpp-public.s3.amazonaws.com/DATASHEET+-+1750+IMU

8. https://gdmissionsystems.com/en/products/communications/spacebornecommunications/spaceborne-gps-receivers/sentinel-m-code-gps-receiver

9. J. Carroll, B. Chan, C. Harner, T. Reid, P. Tarantino, C. Y. T. Yu, ION GNSS, Proceedings of the 24th International Technical Meeting of the Satellite Division of The Institute of Navigation, Portland, OR, 5, pp. 3245-3254 (2011)

10. R.A. Hartunian, G.E. Stewart, S. D. Fergason, T. J. Curtiss, R. W. Seibold, Causes and Mitigation of Radio Frequency (RF) Blackout During Reentry of Reusable Launch Vehicles (John A. Volpe National Transportation Systems Center, Cambridge, MA. Research and Development Administration Transportation Systems Center, 2007)

11. E. G. Lightsey, G. C. Blackburn, J. E. Simpson, GPS WORLD, 11, pp. 30-35 (2000)

12. J. L. Goodman, ION, Proceedings of the 2001 National Technical Meeting of The Institute of Navigation, Long Beach, CA, 1, pp. 709 - 724 (2001)

13. J. Farrell, AIDED NAVIGATION. GPS with High Rate Sensors (McGraw-Hill, Inc. New York, NY, USA, 2008)

14. https:/www.mathworks.com/products/connections/product_detail/satellite-navigationsatnav-toolbox.html

15. K. D. Park, J. Ha, S. Lee, J. of Astr. and Sp. Sc., 27 (2), pp. 117-122 (210)

16. J. A. Klobuchar, IEEE Trans. on Aerosp. and Electr. Sys., 23 (3), pp. 325-331 (1987)

17. T. Schüler, On ground based GPS tropospheric delay estimation (Univ. der Bundeswehr, 2001)

18. E. D. Kaplan, C. J. Hegarty, Understanding GPS. Principles and applications (Artech House, $2^{\text {nd }}$ ed., 2005)

19. P. D. Groves, Principles of GNSS, Inertial, and Multisensor Integrated Navigation Systems (Artech House, $2^{\text {nd }}$ ed., 2013)

20. K. G. Blankinship, IEEE/ION, Position, Location and Navigation Symposium, 1, pp. 1152-1166 (2008)

21. O. J. Woodman, An introduction to inertial navigation (Technical report, University of Cambridge, 2007) 